sequence. The results strongly supported the hypothesized vitamin-controlled structural transition, explaining the observed control of mRNA translation. Similar evidence has been claimed (but not yet published) for an mRNA control region required in vitamin $B_{2}$ biosynthesis.

One puzzle is that the vitamin-sensing mRNA sequences that control translation ${ }^{1,2}$ are considerably larger, at up to 200 nucleotides, than the aptamers that have been generated in vitro, which typically consist of less than 40 nucleotides. Why is this? The most likely explanation is that the mRNA control regions do much more than just bind a target molecule. They function by virtue of their ability to fold into two distinct conformations, which are finely balanced energetically so that a change in vitamin concentration drives the transition from one conformation, which allows translation to begin, to the other, which does not. However, several groups, including Breaker's, have 'evolved' RNA elements in vitro that can switch between conformations in a ligand-dependent way, yet are much smaller than the in vivo examples. Perhaps the large size of the in vivo regulatory sequences is an 'in vivo artefact' of the constraints on the evolution of functional RNA structures from a limited set of initial sequences. In contrast, the in vitro situation generally involves sampling from a large set of random sequences.

Why have metabolite-sensing RNA sequences been found (so far) only in mRNAs involved in vitamin biosynthesis and import? One intriguing possibility, suggested by Winkler et al. ${ }^{1}$, is that these RNA control elements are ancient, dating from the 'RNA world' - a hypothesized early stage in the evolution of life on Earth, when proteins did not exist. All three vitamins in question are biochemically more or less RNA-like ( $B_{12}$ contains an adenosine group), and have themselves been proposed to date from the RNA world ${ }^{7}$. But many other metabolites, and their biosynthetic pathways, must be as old or older. With so much of biochemistry now subject to the domination of proteins, it is unclear why the biosynthesis of these particular metabolites should have remained so staunchly within the realm of RNA. Perhaps these issues will be clarified if and when additional examples of RNA-mediated metabolic control are discovered.

Whatever the answer, it is clear that researchers investigating translational control must bear in mind that non-coding mRNA sequences might regulate this process directly. A different example of this phenomenon was provided recently by Johansson et al. ${ }^{8}$, who described an mRNA regulatory sequence that acts as a direct tem- perature sensor in a pathogenic bacterium, Listeria, controlling translation without needing any regulatory proteins. This RNA sensor detects the increase in temperature that occurs when the bacterium moves into a mammalian host, and regulates the expression of genes associated with bacterial virulence. Along with the discovery of the numerous, widespread micro-RNA molecules, which seem to regulate the translation of mRNAs in a variety of ways, these findings provide striking examples of new and unexpected roles for RNA in controlling gene expression. Given the current pace of discovery, it seems likely that yet more surprises may be just around the corner.

Jack W. Szostak is at the Howard Hughes

Medical Institute, Department of Molecular

Biology, Massachusetts General Hospital,

Boston, Massachusetts 02114, USA.

e-mail: szostak@molbio.mgh.harvard.edu

1. Winkler, W., Nahvi, A. \& Breaker, R. R. Nature 419, 952-956 (2002); advance online publication, 16 October 2002 (doi:10.1038/nature01145).

2. Nahvi, A. et al. Chem. Biol. 9, 1043-1049 (2002).

3. Nou, X. \& Kadner, R. J. Proc. Natl Acad. Sci. USA 97, 9170-9175 (2000)

4. Miranda-Rios, J., Navarro, M. \& Soberón, M. Proc. Natl Acad. Sci. USA 98, 9736-9741 (2001).

Gelfand, M. S. et al. Trends Genet. 15, 439-442 (1999).

6. Stormo, G. D. \& Yongmei, J. Proc. Natl Acad. Sci. USA 98, 9465-9467 (2001).

7. White, H. B. III J. Mol. Evol. 7, 101-104 (1976).

8. Johansson, J. et al. Cell 110, 551-561 (2002).

\title{
Environment
}

\section{Trash trends}

\section{Recycling campaigners and} environmental groups often quote alarming statistics on how much waste one person produces each year. But a comprehensive report on rubbish collected in New York City throughout the twentieth century claims that the figure has dropped dramatically, from its peak in the 1940s.

Writing in Environmental Science and Technology (doi: 10.1021/ es011074t), Daniel Walsh describes the rise and fall of garbage components in the Big Apple. Using the most complete set of municipal records for a US city's residential refuse, Walsh records a maximum output of $940 \mathrm{~kg}$ of waste per person in 1940, and a low of $320 \mathrm{~kg}$ per person in both 1961 and 1963.

Surprisingly, since the 1980s a person's annual throwaways have stabilized at a relatively low $430 \mathrm{~kg}$. Also, the most significant trash trends were mostly declines in the percentages of different categories of waste. The relative amounts of fuel ash, food waste, metal and

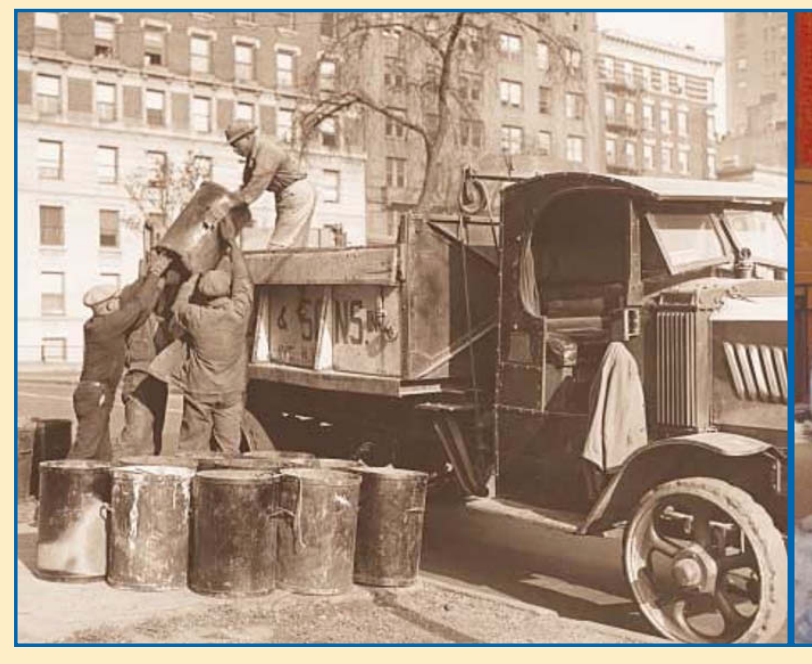

glass dropped, but the percentage of plastics rose and that of paper remained the same. The photograph on the left shows ash collection earlier in the twentieth century; that on the right a present-day scene.

Between 1920 and 1990 there was a $50 \%$ decrease in refuse density. Walsh attributes this to a decrease in coal and other fuel ash, and to technologies that have enabled product packaging to switch from glass and metal to paper and plastic. Over the same period, organic-matter waste rose fourfold, increasing the greenhousegas potential per unit of dumped or incinerated garbage. Walsh estimates that the totality of New York City's refuse for the past

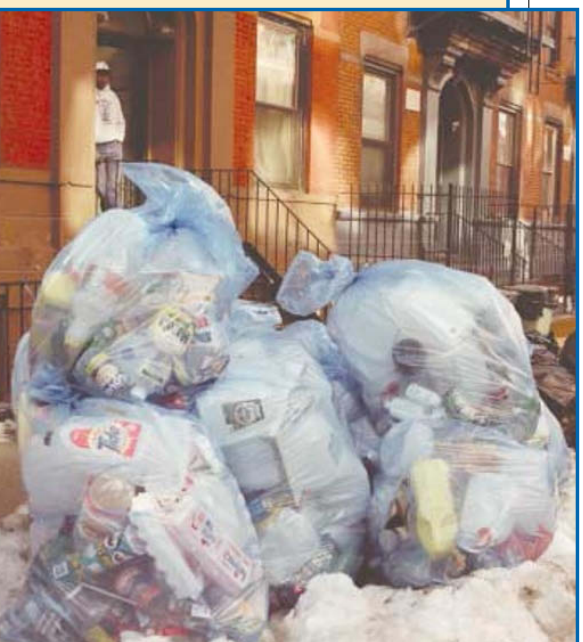

century represents a carbon pool of 80 million tons.

As coal was at the start of the twentieth century, paper is now the most abundant category of refuse, accounting for roughly $35 \%$ of all residential discards. Will the figures for the twenty-first century reflect the long-forecast advent of the paperless office? Kendall Powell 